\title{
REFERENCES FOR APPENDIX
}

\section{Selected Bibliography of Raffaello Caverni}

Caverni, Raffaello. 1874. Problemi naturali di Galileo Galilei e di altri autoridella sua scuola. Firenze: Sansoni.

—. 1877a. De'nuovi studi della filosofia: Discorsi a un giovane studente. Firenze: Carnesecchi.

- 1877b. Voci e modi nella Divina Commedia dell'uso popolare toscano: Dizionarietto. Firenze: Giusti [Reprint: Firenze: Pagnini, 1987].

- 1878. "Notizie storiche intorno all'invenzione del termometro." Bullettino di bibliografia e di storia delle scienze matematiche e fisiche 11:531-86.

- 1879. Dell'arte dello scrivere: Consigli a un giovinetto. Firenze: Le Letture di Famiglia.

—. 1881. Dell'antichità dell'uomo secondo la scienza moderna: Saggio di studi. Firenze: Cellini.

- 1882. Ricreazioni scientifiche. Firenze: Le Letture di Famiglia Editrici.

- 1883. "[Review of] Galileo Galilei e lo Studio di Padova per Antonio Favaro." La Rassegna Nazionale (anno 5) 12:476-80.

- 1884. L'estate in montagna: Nozioni di fisica. Firenze: Le Monnier [Revised edition: 1885].

- 1886a. "[Review of] Carteggio inedito di Ticone Brahe, Giovanni Keplero e di altri celebri astronomi e matematici dei secoli XVI, e XVII con Giovanni Antonio Magini, tratto dall'Archivio Malvezzi de' Medici in Bologna, pubblicato ed illustrato da A. Favaro." La Rassegna Nazionale (anno 8) 28:568-75.

- 1886b. Fra il verde e ifiori: Nozioni di botanica. Firenze: Le Monnier [Second edition: 1900].

- 1888. Con gli occhi per terra: Nozioni intorno alla natura e alle proprietà di alcune sostanze minerali. Firenze: Paggi.

- 1891-1900. Storia del metodo sperimentale in Italia. 6 vols. Firenze: Civelli.

- 1972. Storia del metodo sperimentale in Italia. With an Introductory Note by Giorgio Tabarroni. 6 vols. New York/London: Johnson Reprint Corporation [Reprint of Caverni 1891-1900; other reprint Bologna: Forni, 1970].

\section{Selected Bibliography of Antonio Favaro}

Favaro, Antonio. 1869. Studi sul tracciamento della Galleria delle Alpi Cozie preceduti da cenni storici. Torino: Ceresole e Panizza. 
- 1873. "Beiträge zur Geschichte der Planimeter." Allgemeine Bauzeitung 38:68-90, 93-104.

—. 1874. "Notizie storiche sulle frazioni continue dal secolo decimoterzo al decimosettimo." Bullettino di bibliografia e di storia delle scienze matematiche e fisiche 7:451-502, 533-89.

- 1875. Saggio di cronografia dei matematici dell'antichità. Padova: Sacchetto.

-. 1877. Lezioni di statica grafica. Padova, Sacchetto.

- 1878. "La storia delle matematiche nella Università di Padova." Bullettino di bibliografia e di storia delle scienze matematiche e fisiche 11:799-801.

- 1879-80. "Ragguaglio dei Manoscritti Galileiani nella Biblioteca Nazionale di Firenze ed annuncio di alcuni frammenti inediti di Galileo." Atti del Reale Istituto Veneto di Scienze, Lettere ed Arti 6:847-53.

- 1880. "Le aggiunte autografe di Galileo al Dialogo sopra i due massimi sistemi nell'esemplare posseduto dalla Biblioteca del Seminario di Padova." Atti della Reale Accademia di Scienze, Lettere ed Arti di Modena 19:245-75.

- 1882. "Gli autografi galileiani nell'archivio Marsigli in Bologna." Bullettino di bibliografia e di storia delle scienze matematiche e fisiche 15:581-92.

- 1883. "Alcuni scritti inediti di Galileo Galilei tratti dai manoscritti della Biblioteca Nazionale di Firenze pubblicati ed illustrati." Bullettino di bibliografia e di storia delle scienze matematiche e fisiche 16:1-97, 135-210.

-. [1883] 1966. Galileo Galilei e lo Studio di Padova. 2 vols. Padova: Editrice Antenore [first edition: Firenze: Le Monnier, 1883].

- 1885. "Documenti inediti per la storia dei Manoscritti Galileiani nella Biblioteca Nazionale di Firenze pubblicati ed illustrati." Bullettino di bibliografia e di storia delle scienze matematiche e fisiche 18:1-112, 151-230.

- 1886a. Carteggio inedito di Ticone Brahe, Giovanni Keplero e di altri celebri astronomi e matematici dei secoli XVI e XVII con Giovanni Antonio Magini, tratto dall'Archivio Malvezzi de' Medici in Bologna. Bologna: Zanichelli.

- 1886b. "Intorno ad alcuni documenti galileiani recentemente scoperti nella Biblioteca Nazionale di Firenze." Bullettino di bibliografía e di storia delle scienze matematiche e fisiche 19:1-54.

- 1887a. Miscellanea galileiana inedita. Studi e ricerche. Venezia: Antonelli [also in Memorie del Reale Istituto Veneto di Scienze, Lettere ed Arti 22 (1882): 701-1037].

- $1887 \mathrm{~b}$. "Otto anni d'insegnamento di Storia delle Matematiche nella R. Università di Padova." Bibliotheca Mathematica 1:49-54.

- 1887c. "Di Giovanni Tade e di una sua visita a Galileo dal 12 al 15 novembre 1614." Bullettino di bibliografia e di storia delle scienze matematiche e fisiche 20:345-71.

- 1888. Per la Edizione Nazionale delle opere di Galileo Galilei sotto gli auspicii di S. M. il Re d'ttalia. Esposizione e disegno. Firenze: Barbèra.

- 1891. "Nuovi studi galileiani." Memorie del Reale Istituto Veneto di Scienze, Lettere ed Arti 24:7-430. 
- 1894-95. "Don Baldassarre Boncompagni e la storia delle scienze matematiche e fisiche." Atti del Reale Istituto Veneto di Scienze, Lettere ed Arti 6:509-21. -1898-99. "Intorno alle opere scientifiche di Galileo Galilei nella Edizione Nazionale sotto gli auspicii di S. M. il Re d'Italia." Atti del Reale Istituto Veneto di Scienze, Lettere ed Arti 58 (part 2):129-204.

-1899-1900. "Raffaello Caverni. Nota commemorativa." Atti del Reale Istituto Veneto di Scienze, Lettere ed Arti 59 (part 2):377-79.

- 1907a. "Antichi e moderni detrattori di Galileo." La Rassegna Nazionale (anno 29) 153:577-600.

- 1907b. Galileo e l'Inquisizione. Documenti del processo galileiano esistenti nell'Archivio del S. Uffizio e nell'A rchivio Segreto Vaticano per la prima volta integralmente pubblicati. Firenze: Barbèra [Reprint: Firenze: Giunti-Barbèra, 1983].

- [1907-08] 1983. “Amici e corrispondenti di Galileo Galilei XXI. Benedetto Castelli." Atti del Reale Istituto Veneto di Scienze, Lettere ed Arti 67 (part 2):1-130 [Reprint in Favaro 1983, 2:737-870].

- 1910. Galileo Galilei. Modena: Formiggini. [New edition: Firenze: Barbèra, 1964].

- 1911-12. Atti della Nazione Germanica Artista nello Studio di Padova. 2 vols. Venezia: Tipografia Emiliana.

- 1912-13a. "Amici e corrispondenti di Galileo Galilei XXIX. Vincenzio Viviani." Atti del Reale Istituto Veneto di Scienze, Lettere ed Arti 72 (part 2):1-155 [Reprint in Favaro 1983, 2:1007-163].

- 1912-13b. "Emilio Wohlwill. Nota commemorativa." Atti e Memorie della Reale Accademia di Scienze, Lettere ed Arti in Padova 29:43-55.

- [1913-14] 1992b. "Serie ventesimaterza di Scampoli galileiani CXLVI. Galileo e Guidobaldo del Monte." Atti e Memorie della Reale Accademia di Scienze, Lettere ed Arti in Padova 30:54-61 [Reprint in Favaro 1992b, 2:716-23].

- [1914-15] 1983. "Amici e corrispondenti di Galileo Galilei XXXI. Bonaventura Cavalieri." Atti del Reale Istituto Veneto di Scienze, Lettere ed Arti 74 (part 2):701-67 [Reprint in Favaro 1983, 3:1245-315].

- 1916. "La condanna di Galileo e le sue conseguenze per il progresso degli studi." Scientia anno 10, vol. 20, no. 51:1-11.

- 1916-17. "Amici e corrispondenti di Galileo Galilei XXXVIII. Marino Mersenne." Atti del Reale Istituto Veneto di Scienze, Lettere ed Arti 76 (part 2):35-92 [Reprint in Favaro 1983, 3:1475-534].

- 1917. "Scritture Galileiane apocrife", Bollettino di bibliografia e storia delle scienze matematiche 19:33-43.

- 1917-18. "Intorno alla prima edizione fiorentina delle opere di Galileo." Atti del Reale Istituto Veneto di Scienze, Lettere ed Arti 77 (part 2):229-42.

-. 1918. "Galileo Galilei e $i$ Doctores parisienses." Rendiconti della Reale Accademia dei Lincei. Classe di scienze morali, storiche efilologiche 27:139-50.

-. 1919. "La storia delle scienze e la storia delle università a proposito di un 
prossimo centenario." Atti della Società Italiana per il Progresso delle Scienze 10:457-60.

- [1919-20a] 1992a. "Adversaria galilaeiana. Serie quinta. XXXIII. Scritture Galileiane apocrife." Atti e Memorie della Reale Accademia di Scienze, Lettere ed Arti in Padova 36:17-30 [Reprint in Favaro 1992a, 141-54.]

- 1919-20b. "Galileo Galilei, Benedetto Castelli e la scoperta delle fasi di Venere." Archivio di storia della scienza 1:283-96.

—. 1922a. Saggio di bibliografia dello Studio di Padova [1500-1920]. 2 vols. Venezia: Ferrari.

- 1922b. L'Università di Padova. Venezia: Ferrari.

- 1968. Galileo Galilei a Padova. Ricerche e scoperte, insegnamento, scolari. Padova: Editrice Antenore.

- 1983. Amici e corrispondenti di Galileo. A cura e con una nota introduttiva di Paolo Galluzzi. 3 vols. Firenze: Libreria Editrice Salimbeni.

- 1992a. Adversaria galilaeiana. Serie I-VII. Ristampa anastatica dagli "Atti e Memorie" della Accademia Patavina di Scienze, Lettere ed Arti. Edited by Lucia Rossetti and Maria Laura Soppelsa. Trieste: Edizioni Lint.

- 1992b. Scampoligalileiani. Ristampa anastatica dagli "Atti e Memorie"della Accademia Patavina di Scienze, Lettere ed Arti. Edited by Lucia Rossetti and Maria Laura Soppelsa. 2 vols. Trieste: Edizioni Lint.

Favaro, Antonio et al. [1889-90] 1972. "Relazione della Giunta del R. Istituto Veneto deputata all'esame dei lavori presentati al concorso della Fondazione Tomasoni sul tema: Storia del metodo sperimentale in Italia." Atti del Reale Istituto Veneto di Scienze, Lettere ed Arti serie 7, vol. 1, 319-43 [Reprinted in Caverni 1972, 1:5-20].

Carli, Alarico; Favaro, Antonio. 1896. Bibliografia galileiana (1568-1895). Roma: Libreria dello Stato [Reprint: Bologna: Brighenti, 1972].

\section{Selected Bibliography of Emil Wohlwill}

Wohlwill, Emil. 1863-64. "Bacon von Verulam und die Geschichte der Naturwissenschaft." Deutsche Jahrbücher für Politik und Literatur 9 (1863), 382-415; 10 (1864):207-44.

- 1865. "Zur Geschichte der Erfindung und Verbreitung des Thermometers." Annalen der Physik und Chemie 124:163-78.

- 1866. "Die Entdeckung des Isomorphismus. Eine Studie zur Geschichte der Chemie." Zeitschrift für Völkerpsychologie und Sprachwissenschaft 4:1-67.

—. 1867. "Vorlesungen zur Geschichte der Wiederbelebung der Naturwissenschaften im 16. Jahrhundert." Lecture by Dr. Emil Wohlwill in the great auditorium of the Johanneum, January through March (unpublished).

-1870. Der Inquisitionsprocess des Galileo Galilei. Eine Prüfung seiner rechtlichen Grundlage nach den Acten der Römischen Inquisition. Berlin: R. Oppenheim. 
1872a. "Zum Inquisitionsprocess des Galileo Galilei." Literaturzeitung der Zeitschrift für Mathematik und Physik 17:9-31.

- 1872b. "Erwiederung. Zum Inquisitionsprocess des Galileo Galilei." Literaturzeitung der Zeitschrift für Mathematik und Physik 17:81-98.

—. 1877a. Ist Galilei gefoltert worden? Eine kritische Studie. Leipzig: Duncker \& Humblot.

—. 1877b. "Die Fälschung des Protokolls vom 26. Februar 1616."(unpublished; Niedersächsische Staats- und Universitätsbibliothek Göttingen, Hist. lit. biogr. II. 6249).

- 1879. "Der Original-Wortlaut des päpstlichen Urtheils gegen Galilei." Historisch-literarische Abtheilung der Zeitschrift für Mathematik und Physik 24:1-26.

- 1880. "Erklärung und Abwehr." Historisch-literarische Abtheilung der Zeitschrift für Mathematik und Physik 25:185-90.

-1 1883. "[Review of] Antonio Favaro, Galileo Galilei e lo Studio di Padova. Florenz, Le Monnier, 1883." Deutsche Literaturzeitung 4:204-06.

—. 1883-84. "Die Entdeckung des Beharrungsgesetzes." Zeitschrift für Völkerpsychologie und Sprachwissenschaft 14 (1883):365-410; 15 (1884):70-135, $337-87$.

—.1887a. "Die Prager Ausgabe des Nuncius sidereus." Bibliotheca Mathematica $1: 100-02$.

—. 1887b. "Joachim Jungius und die Erneuerung atomistischer Lehren im 17. Jahrhundert. Ein Beitrag zur Geschichte der Naturwissenschaften in Hamburg." In Festschrift zur Feier des fünfzigjährigen Bestehens des Naturwissenschaftlichen Vereins in Hamburg, 18. November 1887 [= Abhandlungen aus dem Gebiete der Natuwissenschaften, vol. 10], 1-66. Hamburg: Friederichsen \& Co.

- 1888a. Joachim Jungius. Festrede zur Feier seines dreihundersten Geburtstages am 22. Oktober 1887. Mit Beiträgen zu Jungius' Biographie und zur Kenntnis seines handschriftlichen Nachlasses. Hamburg/Leipzig: Voss.

—. 1888b. "Hat Leonardo da Vinci das Beharrungsgesetz gekannt?" Bibliotheca Mathematica 2:19-26.

- 1889. "[Review of] Antonio Favaro: Per la edizione nazionale delle opere di Galileo Galilei. Esposizione e disegno. Florenz, Barbèra, 1888." Deutsche Literaturzeitung 10:129-31.

- 1891a. "[Review of] Le Opere di Galileo Galilei. Edizione Nazionale. Direttore: Antonio Favaro. Vol. I. Florenz, Barbèra, 1890." Deutsche Literaturzeitung 12:823-25.

- 1891b. "[Review of] Antonio Favaro, Galileo Galilei e Suor Maria Celeste. Florenz, Barbèra, 1891." Deutsche Literaturzeitung 12:825-27.

- 1891 c. "[Review of] Anton von Braunmühl, Christoph Scheiner als Mathematiker, Physiker und Astronom. Bamberg, Buchner, 1891." Deutsche Literaturzeitung 12:1247-249.

-. 1894. "Galilei betreffende Handschriften der Hamburger Stadtbibliothek." 
Jahrbuch der Hamburgischen Wissenschaftlichen Anstalten 12:147-223. [Also published as a monography, Hamburg: Gräfe \& Sillem, 1895]

—. 1895. “[Review of] Laurenz Müllner, Die Bedeutung Galilei’s für die Philosophie. Wien, Selbstverlag der k. k. Universität, 1894." Deutsche Literaturzeitung 16:1462-467.

—. 1899. "Die Entdeckung der Parabelform der Wurflinie." Abhandlungen zur Geschichte der Mathematik 9(1899) [= Zeitschrift für Mathematik und Physik 44 (1899), Supplement], 577-624.

- 1902. "Neue Beiträge zur Vorgeschichte des Thermometers." Mitteilungen zur Geschichte der Medizin und der Naturwissenschaften 1:5-8, 57-62, 143-58, 282-90.

—. 1904a. "Über einen Grundfehler aller neueren Galileibiographien." In Verhandlungen der Gesellschaft Deutscher Naturforscher und Ärzte. 75. Versammlung zu Cassel, 20.-26. September 1903. Zweiter Teil, 2. Hälfte, 100-01. Leipzig: Vogel.

—. 1904b. "Melanchthon und Copernicus." Mitteilungen zur Geschichte der Medizin und der Naturwissenschaften 3:260-67.

—. 1905. "Galilei-Studien. I. Die Pisaner Fallversuche." Mitteilungen zur Geschichte der Medizin und der Naturwissenschaften 4:229-48.

—_. 1906a. "Ein Vorgänger Galileis im 6. Jahrhundert." Physikalische Zeitschrift $7: 23-32$.

-. 1906b. "Galilei-Studien. II. Der Abschied von Pisa." Mitteilungen zur Geschichte der Medizin und der Naturwissenschaften 5:230-49, 439-64.

—. 1907. "Galilei-Studien. II. Der Abschied von Pisa (Zweites Nachwort)." Mitteilungen zur Geschichte der Medizin und der Naturwissenschaften 6:231-42.

—. 1909. "Zur Geschichte der Entdeckung der Sonnenflecken." Archiv für die Geschichte der Naturwissenschaften und der Technik 1:443-54.

-. 1909-29. Galilei und sein Kampf für die Copernicanische Lehre. 2 vols. Hamburg/Leipzig: Voss.

- 1912. "Naturforscher als Historiker der Naturwissenschaften." Mitteilungen zur Geschichte der Medizin und der Naturwissenschaften 11:1-5.

—. 1929. "Für eine Veröffentlichung von Jungius' Werken und seine künftige Biographie." In Beiträge zur Jungius-Forschung. Prolegomena zu der von der Hamburgischen Universität beschlossenen Ausgabe der Werke von Joachim Jungius (1587-1657), edited by Adolf Meyer, 15-20. Hamburg: Hartung.

- 1969. Galilei und sein Kampf für die Copernicanische Lehre. With an Introductory Note by Hans-Werner Schütt. 2 vols. Wiesbaden: Sändig [Reprint of Wohlwill 1909-29]. 


\section{Other Works}

Baldini, Ugo. 1980. "La scuola galileiana." In Storia d'Italia. Annali 3: Scienza e tecnica nella cultura e nella società dal Rinascimento a oggi, edited by Gianni Micheli, 381-463. Torino: Einaudi.

Baldo Ceolin, Milla; Olivieri, Luigi. 1994-95. "Progetto di edizione degli Studi Galileiani di A. Favaro." Atti e Memorie dell'Accademia Patavina di Scienze, Lettere ed Arti (Parte 3) 107:273-84.

Betti, Umberto; Pagnini, Gian Piero (eds.) 1991. Raffaello Caverni 1837-1900. Antologia degli scritti a cura di Umberto Betti. Note biografiche, storicogenealogiche di Gian Piero Pagnini. Firenze: Pagnini.

Bortolotti, Ettore. 1923-24. "Antonio Favaro storico delle scienze matematiche." Atti e Memorie della Regia Deputazione di Storia Patria per le Provincie di Romagna 14:1-24.

Bosmans, Henri. 1923. "Antonio Favaro (1847-1922)." Revue des questions scientifiques 83:156-75.

Brugnaro, Francesco Giovanni. 1979. "Antonio Favaro, studioso di Galileo Galilei." In Medioevo e Rinascimento veneto con altri studi in onore di Lino Lazzarini. vol. II: Dal Cinquecento al Novecento, 289-306. Padova: Editrice Antenore.

Bucciantini, Massimo. 1986. "Bibliografia e storia della scienza in Italia (1868-1920)." In Biblioteche speciali, edited by Mauro Guerrini, 110-134. Milano: Editrice Bibliografica.

—. 1995. "Favaro, Antonio." Dizionario biografico degli italiani 45:441-44. Roma: Istituto della Enciclopedia Italiana.

Campori, Giuseppe (ed.). 1881. Carteggio Galileano inedito, con note ed appendici. Modena: Società Tipografica Antica Tipografia Soliani $[=$ Memorie della Regia Accademia di Scienze, Lettere ed Arti in Modena 20 (1880-82), part 2].

Cappelletti, Vincenzo. 1969. "Boncompagni Ludovisi, Baldassarre." Dizionario biografico degli italiani 1 1:704-09. Roma: Istituto della Enciclopedia Italiana.

Cappelletti, Vincenzo; Di Trocchio, Federico. 1979. "Caverni, Raffaello." Dizionario biografico degli Italiani 23:85-8. Roma: Istituto della Enciclopedia Italiana.

Cavalieri, Bonaventura. 1632. Lo Specchio ustorio overo Trattato delle settioni coniche, et alcuni loro mirabili effetti intorno al lume, caldo, freddo, suono e moto ancora. Bologna: Ferroni [Second edition: Bologna, Ferroni, 1650].

Collingwood, R. G. [1946] 1993. The Idea of History. Revised Edition with Lectures 1926-1928. Edited with an Introduction by Jan van der Dussen. Oxford: Clarendon Press.

Damerow, Peter; Jürgen Renn, Simone Rieger. 1996. Pilot Study for a Systematic PIXE Analysis of the Ink Types in Galileo's Ms. 72. Project Report No. I. Berlin: Max-Planck-Institut für Wissenschaftsgeschichte [= Max-PlanckInstitut für Wissenschaftsgeschichte, Preprint 54]. 
Del Lungo, Carlo. 1919. "Sopra la Storia del metodo sperimentale in Italia di R. Caverni." Atti della Società Italiana per il Progresso delle Scienze 10:477-78.

- 1919-20. "La Storia del metodo sperimentale in Italia di Raffaello Caverni." Archivio di storia della scienza 1:272-82.

_- 1921-22. "Del pendolo e della sua applicazione all'orologio." Archivio di storia della scienza 2:147-66.

Descartes, René. 1897-1913. Oeuvres. Edited by Charles Adam and Paul Tannery. Paris: Cerf.

Drake, Stillman. 1979. Galileo's Notes on Motion Arranged in Probable Order of Composition and Presented in Reduced Facsimile. Firenze: Istituto e Museo di Storia della Scienza.

Duhem, Pierre. 1909. "Sur la mécanique de Léonard de Vinci et les recherches de Raffaello Caverni." In Pierre Duhem, Études sur Léonard de Vinci. Ceux qu'il a lus et ceux qui l'ont lu, vol. 2, 361-63. Paris: Hermann [Reprint: Paris: Éditions des Archives Contemporaines, 1984].

Favaro, Giuseppe. 1922-23. "Antonio Favaro. Bio-bibliografia." Atti del Reale Istituto Veneto di Scienze, Lettere ed Arti 82 (part 1):221-303.

Gabrieli, Giuseppe. 1925. "A. Favaro e gli studi italiani di storia della scienza." Isis 7:456-67.

Galilei, Galileo. 1632. Dialogo dove ne i congressi di quattro giornate si discorre sopra $i$ due massimi sistemi del mondo tolemaico, e copernicano. Firenze: Landini. [Reprinted in Galilei 1890-1909, 7:21-520.]

- 1638. Discorsi e dimostrazioni matematiche, intorno à due nuove scienze attenenti alla mecanica et $i$ movimenti locali. Con una appendice del centro di gravità d'alcuni solidi. Leida: Elsevirii. [Reprinted in Galilei 1890-1909, 8:39-318.]

—. 1718. Opere. Nuova edizione coll'aggiunta di varj trattati dell istesso autore non più dati alle stampe. Edited by Tommaso Buonaventuri et al. 3 vols. Firenze: Tartini e Franchi.

- 1842-56. Le Opere. Prima edizione completa condotta sugli autentici manoscritti Palatini. Edited by Eugenio Albèri et al. 16 vols. Firenze: Società Editrice Fiorentina.

-1890-1909. Le Opere. Edizione nazionale sotto gli auspici di Sua Maestà il Re d'Italia. Edited by Antonio Favaro et al. 20 vols. Firenze: Barbèra [Third edition: Firenze: Barbèra, 1968].

- 1891. Dialog über die beiden hauptsächlichsten Weltsysteme, das ptolemäische und das kopernikanische. Aus dem Italienischen übersetzt und erläutert von Emil Strauss. Leipzig: Teubner.

- 1974. Two New Sciences Including Centers of Gravity and Force of Percussion. Translated with introduction and notes by Stillman Drake. Madison, Wisconsin: University of Wisconsin Press.

Galluzzi, Paolo. 1983. "[Introductory Note]." In Favaro 1983, 1:V-XII. 
Galluzzi, Paolo, et al. 1988. Galileo: La sensata esperienza. Cinisello Balsamo (Milano): Pizzi.

Giovannozzi, Giovanni. 1910. “Un tedesco di Montelupo.” La Rassegna Nazionale (anno 32) 171:25-74.

- 1920. "Raffaello Caverni e la sua Storia del metodo sperimentale." Archivio di storia della scienza 1:266-71.

- 1928. "Un capitolo inedito della Storia del metodo sperimentale in Italia." Memorie della Pontificia Accademia delle Scienze. I Nuovi Lincei 11:171-90.

Henneberg, Lebrecht. 1901-08. "Die graphische Statik der starren Körper." In Mechanik. vol. 1 (= Encyklopädie der mathematischen Wissenschaften mit Einschluss ihrer Anwendungen, 4, 1), edited by Felix Klein and Conrad Müller, 345-434. Leipzig: Teubner.

Huygens, Christiaan. 1888-1950. Oeuvres complètes. Publiées par la Société Hollandaise des Sciences. La Haye: Nijhoff.

Klug, Josef. 1913. "Die nachgelassenen Schriften Dr. Emil Wohlwills." Archiv für die Geschichte der Naturwissenschaften und der Technik 6:216-21.

Laemmel, Rudolf. 1928. "Untersuchung der Dokumente des Galileischen Inquisitionsprozesses." Archiv für Geschichte der Mathematik, der Naturwissenschaften und der Technik 10:405-19.

Landucci, Giovanni. 1996. “Filosofia sperimentale'e immagini di Galileo nell'Ottocento italiano." In Handjaras, Luciano, et al. Ricerche di filosofia: tra ermeneutica e filosofia analitica, 151-207. Firenze: Alfani.

Lefons, Chiara. 1984. "Un capitolo dimenticato della storia delle scienze in Italia: Il «Bullettino di bibliografia e di storia delle scienze matematiche e fisiche» di Baldassarre Boncompagni." Giornale critico della filosofia italiana 63:65-90.

Libri, Guillaume. 1838-41. Histoire des sciences mathématiques en Italie, depuis la renaissance des lettres jusqu'à la fin du dix-septième siècle. 4 vols. Paris: Renouard [Reprint: Sala Bolognese (Bologna): Forni, 1991].

Maffioli, Cesare S. 1985. "Sulla genesi e sugli inediti della Storia del metodo sperimentale in Italia di Raffaello Caverni." Annali dell'Istituto e Museo di Storia della Scienza di Firenze 10:23-85.

Malusa, Luciano. 1977. "Storiografia filosofica e storiografia scientifica in Antonio Favaro." In Malusa, Luciano. La storiografia filosofica italiana nella seconda metà dell'Ottocento. vol. 1: Tra positivismo e neokantismo, 551-66. Milano: Marzorati.

Martini, Tito. 1901. "Raffaello Caverni e la sua Storia del metodo sperimentale in Italia." L'Ateneo Veneto (anno 24) 1:291-321.

Micheli, Gianni. 1980. "Scienza e filosofia da Vico a oggi." In Storia d'Italia. Annali 3: Scienza e tecnica nella cultura e nella società dal Rinascimento a oggi, edited by Gianni Micheli, 549-675. Torino: Einaudi.

_. 1987. "La storia della scienza nella cultura italiana." In La scienza tra filosofia e storia in Italia nel Novecento, edited by Fabio Minazzi and Luigi Zanzi, 
295-308. Roma: Presidenza del Consiglio dei Ministri. Direzione generale delle informazioni e della proprietà letteraria, artistica e scientifica.

—. 1988. "L'idea di Galileo nella cultura italiana dal XVI al XIX secolo." In Galluzzi et al. 1988, 163-86.

Mieli, Aldo. 1919-20. "L'opera di Raffaello Caverni come storico (cenni preliminari)." Archivio di storia della scienza 1:262-65.

Procacci, P. 1900. "P. Raffaello Caverni." La Rassegna Nazionale (anno 22) 111:804-05.

Quaranta, Mario. 1983. "Antonio Favaro (1847-1922)." In Galileo e Padova: Mostra di strumenti, libri, incisioni, 52-55. Padova: Comune di Padova, Assessorato ai beni culturali.

Renn, Jürgen, et al. 1998. Hunting the White Elephant. When and How Did Galileo Discover the Law of Fall? Berlin: Max-Planck-Institut für Wissenschaftsgeschichte [= Max-Planck-Institut für Wissenschaftsgeschichte, Preprint 97].

Schiaparelli, Giovanni. [1892] 1930. "La Storia del metodo sperimentale in Italia. Cenno bibliografico." Il Pensiero italiano (anno 2) 4(1892):405-30 [Reprinted in Schiaparelli, Giovanni V. Le Opere. 10:3-23. Milano: Hoepli, 1930. Other Reprint: New York, Johnson Reprint Corporation, 1968].

Schütt, Hans-Werner. 1969. "Emil Wohlwill, Galilei und sein Kampf für die Copernicanische Lehre." In Wohlwill 1969, vol. 1, without pagination.

Segre, Michael. 1991. In the Wake of Galileo. New Brunswick (New Jersey): Rutgers University Press.

Seneca, Federico. 1967. Antonio Favaro, Isidoro Del Lungo el'edizione nazionale delle opere galileiane. Firenze: Barbèra.

- 1995 "Antonio Favaro, studioso di Galileo." In Galileo Galilei e la cultura veneziana, 381-404. Venezia: Istituto Veneto di Scienze, Lettere ed Arti.

Strappini, Lucia. 1990. "Del Lungo, Isidoro." Dizionario biografico degli italiani 38:96-100. Roma: Istituto della Enciclopedia Italiara.

Strauss, Emil. 1891. "Einleitung" in Galilei 1891, VI-LXXIX.

Tabarroni, Giorgio. 1969. "Raffaello Caverni: prete contestatore. A 70 anni dalla morte." Physis 11:564-70.

—. 1972. "Raffaello Caverni and his Work: An Introductory Note." In Caverni 1972, 1:v-xxii.

Tannery, Paul. 1900. "[Review of] Emil Wohlwill. Die Entdeckung der Parabelform der Wurflinie. Abhandlungen zur Geschichte der Mathematik, IX, p. 579-635, Teubner, Leipzig; 1899." Bulletin des sciences mathématiques 24:33-7 [Reprinted in: Tannery, Paul. Mémoires scientifiques. Edited by J.-L. Heiberg and H.-G. Zeuthen. 12:157-61. Paris: Gauthier-Villars, 1933].

Tartaglia, Niccolò. 1537. Nova scientia. Vinegia: Stephano da Sebio.

Timpanaro, Sebastiano. 1957. "Storia del metodo sperimentale in Italia." In Dizionario Letterario Bompiani delle opere e dei personaggi di tutti i tempi e di tutte le letterature. Opere. 7:164-65. Milano: Bompiani. 
Torricelli, Evangelista. 1644. Opera geometrica. Florentiae: Typis Amatoris Massae \& Laurentij de Landis.

- 1919-1944. Opere. Edited by Gino Loria and Giuseppe Vassurra. vols. 1-3: Faenza: Montanari, 1919; vol. 4: Faenza: Lega, 1944.

Viviani, Vincenzio. 1674. Quinto libro degli Elementi d'Euclide ovvero Scienza universale delle proporzioni spiegata colla dottrina del Galileo. Firenze: Alla Condotta.

Wisan, Winifred Lovell. 1974. "The New Science of Motion: A Study of Galileo's De motu locali." Archive for History of Exact Sciences 13:103-306. 